\title{
Effective thermal conductivity of polycrystalline materials with randomly oriented superlattice grains
}

\author{
Fan Yang, ${ }^{1}$ Teruyuki Ikeda, ${ }^{2,3}$ G. Jeffrey Snyder, ${ }^{2}$ and Chris Dames ${ }^{1, a)}$ \\ ${ }^{1}$ Department of Mechanical Engineering, University of California at Riverside, California 92521, USA \\ ${ }^{2}$ Materials Science, California Institute of Technology, 1200 E. Colorado Boulevard, Pasadena, \\ California 91125, USA \\ ${ }^{3}$ PRESTO, Japan Science and Technology Agency, 4-1-8 Honcho, Kawaguchi, Saitama 332-0012, Japan
}

(Received 29 April 2010; accepted 29 May 2010; published online 5 August 2010)

\begin{abstract}
A model has been established for the effective thermal conductivity of a bulk polycrystal made of randomly oriented superlattice grains with anisotropic thermal conductivity. The in-plane and cross-plane thermal conductivities of each superlattice grain are combined using an analytical averaging rule that is verified using finite element methods. The superlattice conductivities are calculated using frequency dependent solutions of the Boltzmann transport equation, which capture greater thermal conductivity reductions as compared to the simpler gray medium approximation. The model is applied to a $\mathrm{PbTe} / \mathrm{Sb}_{2} \mathrm{Te}_{3}$ nanobulk material to investigate the effects of period, specularity, and temperature. The calculations show that the effective thermal conductivity of the polycrystal is most sensitive to the in-plane conductivity of each superlattice grain, which is generally four to five times larger than the cross-plane conductivity of a grain. The model is compared to experimental measurements of the same system for periods ranging from 287 to 1590 $\mathrm{nm}$ and temperatures from 300 to $500 \mathrm{~K}$. The comparison suggests that the effective specularity increases with increasing annealing temperature and shows that these samples are in a mixed regime where both Umklapp and boundary scattering are important. () 2010 American Institute of Physics. [doi:10.1063/1.3457334]
\end{abstract}

\section{INTRODUCTION}

The effective thermal conductivity of a superlattice can be greatly reduced as compared to the bulk thermal conductivities of its constituent materials. Although this thermal conductivity reduction is detrimental for applications such as semiconductor lasers, it is advantageous for thermoelectric energy conversion, resulting in large increases in the thermoelectric performance of superlattices in both the in-plane ${ }^{1}$ and cross-plane ${ }^{2}$ directions. Although for very-short-period superlattices phonon wave interference effects may be important, ${ }^{3,4}$ to a large extent the thermal conductivity reductions in superlattices can be understood through the increased scattering rate of phonon particles at boundaries and interfaces., ${ }^{5,6}$

The large majority of superlattice materials that have been experimentally studied to date were synthesized using molecular beam epitaxy (MBE) or metal-organic chemical vapor deposition (MOCVD). ${ }^{1,2} \mathrm{MBE}$ and MOCVD provide extraordinary control over the materials selection, interface quality, and layer thicknesses, but it is difficult to scale these methods up to synthesize the large quantities of material that would be necessary for widespread commercial applications. Therefore, much recent activity has been directed toward low-cost synthesis strategies to create bulk-scale samples that naturally contain internal nanostructures to provide similar levels of phonon scattering as seen in the MBE and MOCVD superlattices. Several examples of this "nanobulk" approach $^{7}$ are the use of reduced grain sizes (such as hot

${ }^{a)}$ Electronic mail: cdames@ucr.edu. pressed nanopowders of $\left.\mathrm{Bi}_{\mathrm{x}} \mathrm{Sb}_{2-\mathrm{x}} \mathrm{Te}_{3}\right)^{8-10}$ as well as complex crystal structures (such as skutterudites, clathrates, and Zintl phases), ${ }^{11}$ and self-assembled composites [such as precipitates ${ }^{12}$ or lamellae of $\mathrm{PbTe} / \mathrm{Sb}_{2} \mathrm{Te}_{3}$ (Ref. 13)]. Many of these nanobulk materials can be considered approximately as made up of numerous randomly-oriented grains, and in many of these materials systems the thermal conductivity within a single grain is expected to be highly anisotropic, due to the anisotropy of the constituent materials ${ }^{8}$ and/or nanoscale superlattice layering within each grain ${ }^{13}$ [Fig. 1(a)].

Motivated by materials systems such as the selfassembled $\mathrm{PbTe} / \mathrm{Sb}_{2} \mathrm{Te}_{3}$ nanocomposite of Ikeda et al., ${ }^{11,13}$ the primary objective of this paper is to model the effective macroscopic thermal conductivity $\kappa_{\text {eff }}$ of bulk polycrystalline materials made up of randomly-oriented superlattice grains. This paper is organized as follows. In Sec. II we use analytical and numerical methods to establish the averaging rule for

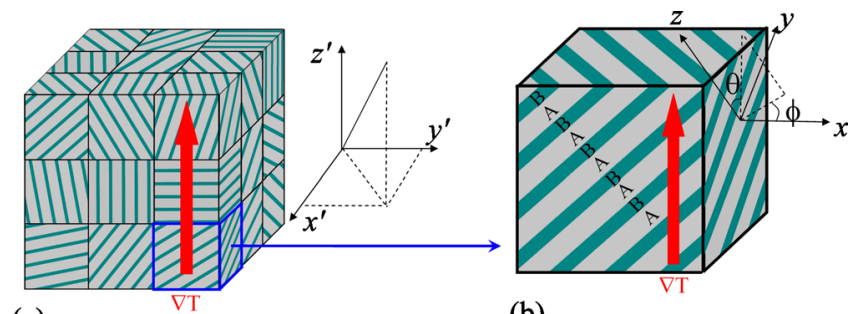

(a)

(b)

FIG. 1. (Color online) (a) Schematic of a nanobulk composite material made of randomly oriented superlattice grains, and the global coordinate system $x^{\prime} y^{\prime} z^{\prime}$, which is aligned to the macroscopic temperature gradient. (b) A single superlattice grain with its local coordinate system $x y z$, which is aligned to the superlattice planes. 
$\kappa_{\text {eff }}$ as a function of the in-plane $\left(\kappa_{x}\right)$ and cross-plane $\left(\kappa_{z}\right)$ thermal conductivities of a single superlattice grain. In Sec. III we model $\kappa_{x}$ and $\kappa_{z}$ themselves, by extending established solutions of the Boltzmann transport equation (BTE) (Refs. 5,6 , and 14) to explicitly account for the frequency dependence of phonon velocities and scattering rates. Then in Sec. IV results are presented for a model $\mathrm{PbTe} / \mathrm{Sb}_{2} \mathrm{Te}_{3}$ nanobulk material, including the effects of period, interface specularity, temperature, and gray versus frequency-dependent modeling. Finally, in Sec. V the calculations are compared to experimental measurements for the same system.

\section{AVERAGING RULES FOR THE EFFECTIVE THERMAL CONDUCTIVITY}

\section{A. General considerations}

We focus on materials such as the self-assembled $\mathrm{PbTe} / \mathrm{Sb}_{2} \mathrm{Te}_{3}$ lamellar structures reported in Ref. 13, which consist of numerous grains, each of which contains many ( 10-100) superlattice periods. We assume that every grain has equal probability to be oriented in any direction. Furthermore we neglect the effect of contact resistances between various grains because the typical grain size is much larger than the superlattice period, and because the grains are selfassembled implying excellent thermal contact between adjacent grains. Within each individual grain, we define a local coordinate system with the local $z$-axis aligned perpendicular to the superlattice planes, and the local $x$ - and $y$-axes aligned parallel to the superlattice planes [Fig. 1(b)].

We restrict the analysis to materials systems where the transport properties in the local $x$ and $y$ directions within a single grain are identical, which is equivalent to assuming that the effective thermal conductivity tensor within each grain, $\mathbf{K}$, has hexagonal, tetragonal, or trigonal symmetries. ${ }^{15}$ This restriction is appropriate for the large majority of practical superlattice materials systems. For example, this restriction always applies to superlattice constituent materials with cubic symmetries (such as $\mathrm{Si}, \mathrm{Ge}, \mathrm{GaAs}$, and $\mathrm{PbTe}$ ), regardless of the layer orientation; and it also applies for constituent materials with layered unit cells (such as graphite, $\mathrm{Bi}_{2} \mathrm{Te}_{3}, \mathrm{Sb}_{2} \mathrm{Te}_{3}$, and $\mathrm{WSe}_{2}$ ) provided that the high-symmetry (c)-axis of the unit cell is aligned normal to the interfacial "habit planes" between adjacent superlattice layers, as is commonly the case. ${ }^{2,16}$ This restriction also holds in the special (nonsuperlattice) case of a single-phase system as long as the constituent material has the required hexagonal, tetragonal, or trigonal symmetry, such as nanocrystalline $\mathrm{Bi}_{\mathrm{x}} \mathrm{Sb}_{2-\mathrm{x}} \mathrm{Te}_{3}$ (Refs. 8-10) or polycrystalline graphite.

Thus, for the restricted class of symmetries of interest in this work, the principal axes of the effective thermal conductivity tensor within each grain, $\mathbf{K}$, will always be aligned to the $x, y, z$ directions. Referred to the local coordinate system, the thermal conductivity tensor of a single grain can therefore be written

$$
\mathbf{K}=\left(\begin{array}{ccc}
\kappa_{x} & 0 & 0 \\
0 & \kappa_{x} & 0 \\
0 & 0 & \kappa_{z}
\end{array}\right)
$$

\section{B. Averaging rule for a thin film}

Before discussing the nanobulk system of Fig. 1, we first consider a thin polycrystalline film, one grain thick but many grains in width and length, sandwiched between isothermal contacts. According to Fourier's law of heat conduction, the local heat flux is given by

$$
\mathbf{q}=-\mathbf{K} \nabla T=-\mathbf{K} \hat{\mathbf{s}}\|\nabla T\|,
$$

where $\mathbf{q}$ is the heat flux vector and $\nabla T$ is the temperature gradient vector, which has magnitude $\|\nabla T\|$ and points in the $\hat{\mathbf{s}}$ direction. We also use $\hat{\mathbf{s}}$ to define the $\hat{\mathbf{z}}^{\prime}$ direction of the global $x^{\prime} y^{\prime} z^{\prime}$ coordinate system. Therefore, as shown in Fig. $1(\mathrm{~b})$, in the local coordinates of a single grain we have

$$
\hat{\mathbf{s}}=\left(\begin{array}{c}
\sin \theta \cos \phi \\
\sin \theta \sin \phi \\
\cos \theta
\end{array}\right) \text {, }
$$

where $\theta$ and $\phi$ describe the rotation of the grain's coordinate system away from the imposed $\nabla T$.

For a polycrystalline film one grain thick sandwiched between isothermal contacts, all the grains are thermally in parallel and experience the same $\nabla T$. On average it is only the component of $\mathbf{q}$ that is aligned with $\nabla T$ that contributes to the net heat flux. Thus we are interested in the average value of the scalar

$$
q_{\text {net }}=\mathbf{q} \cdot \hat{\mathbf{s}} .
$$

For the $\mathbf{K}$ tensor given in Eq. (1), combining Eqs. (3) and (4) yields

$$
q_{\text {net }}=-\left(\kappa_{x} \sin ^{2} \theta+\kappa_{z} \cos ^{2} \theta\right)\|\nabla T\| .
$$

Comparing with the isotropic form of Fourier's law of heat conduction,

$$
q_{\text {net }}=-\kappa_{\text {eff }}\|\nabla T\|,
$$

for this single grain we identify the effective thermal conductivity as $\kappa_{x} \sin ^{2} \theta+\kappa_{z} \cos ^{2} \theta$. Finally, to obtain the effective thermal conductivity for the entire film, we assume each grain is randomly oriented and average Eq. (5) over all directions

$$
\begin{aligned}
\kappa_{\text {eff,film }} & =\frac{1}{4 \pi} \int_{0}^{4 \pi} \kappa_{\mathrm{eff}} d \Omega \\
& =\int_{0}^{\pi} \frac{1}{2}\left(\kappa_{x} \sin ^{2} \theta+\kappa_{z} \cos ^{2} \theta\right) \sin \theta d \theta
\end{aligned}
$$

where $\Omega$ is the solid angle, and in the last expression of Eq. (7) we have already integrated over $\phi$. Carrying out the $\theta$ integration yields simply

$$
\kappa_{\text {eff,film }}=\frac{2}{3} \kappa_{x}+\frac{1}{3} \kappa_{z} .
$$

It will prove convenient to represent these results in dimensionless form. We define

$$
r=\kappa_{z} / \kappa_{x},
$$

as a thermal conductivity contrast parameter, and 
TABLE I. Parameters used in FEM simulations.

\begin{tabular}{|c|c|c|c|}
\hline & Thin film & Thin wire & Nanobulk \\
\hline Grain Configuration $\left(N_{x} \times N_{y} \times N_{z}\right)$ & $6 \times 6 \times 1$ & $1 \times 1 \times 20$ & $4 \times 4 \times 4$ (most runs); $5 \times 5 \times 5$ (several runs) \\
\hline Grain Size $\left(L_{x} \times L_{y} \times L_{z}\right)$ & $10 \times 10 \times 0.1$ & $1 \times 1 \times 10$ & $1 \times 1 \times 1$ \\
\hline Boundary Conditions & $\begin{array}{c}\text { Constant } T \text { ( } z \text { faces }) ; \text { periodic } \\
\text { ( } x \text { and } y \text { faces })\end{array}$ & $\begin{array}{c}\text { Constant } T \text { ( } z \text { faces }) \\
\text { adiabatic ( } x \text { and } y \text { faces })\end{array}$ & $\begin{array}{c}\text { Periodic with fixed } T \text { difference }(z \text { faces }) \\
\text { periodic ( } x \text { and } y \text { faces })\end{array}$ \\
\hline Equation for comparison & Eq. (11) & Eq. (15) & Eq. (18) \\
\hline
\end{tabular}

$$
\kappa_{\text {char }}=\left(\kappa_{x}^{2} \kappa_{z}\right)^{1 / 3},
$$

as a characteristic thermal conductivity. Thus, Eq. (8) can be expressed

$$
\kappa_{\text {eff,film }} / \kappa_{\text {char }}=\frac{2}{3} r^{-1 / 3}+\frac{1}{3} r^{2 / 3} .
$$

\section{Averaging rule for a long wire}

We now consider a polycrystalline wire one grain in diameter and many grains long. In this case every grain will experience the same heat flux, but now with different $\|\nabla T\|$. In other words, now $\mathbf{q}$ is the forcing and $\nabla T$ is the response. We write

$$
\nabla T=-\mathbf{K}^{-1} \mathbf{q}=-\mathbf{K}^{-1} \hat{\mathbf{s}}\|\mathbf{q}\|,
$$

where now $\hat{\mathbf{s}}$ refers to the direction of the average heat flux. Proceeding in analogy to Eqs. (2)-(8), we take the component of $\nabla T$ along the $\hat{\mathbf{s}}$ direction,

$$
\|\nabla T\|_{\text {net }}=\left(-\mathbf{K}^{-1} \hat{\mathbf{s}}\|\mathbf{q}\|\right) \cdot \hat{\mathbf{s}},
$$

which after averaging over $\Omega$ gives

$$
\kappa_{\text {eff,wire }}=\left(\frac{2}{3} \kappa_{x}^{-1}+\frac{1}{3} \kappa_{z}^{-1}\right)^{-1},
$$

or in dimensionless form,

$$
\kappa_{\text {eff,wire }} / \kappa_{\text {char }}=\left(\frac{2}{3} r^{1 / 3}+\frac{1}{3} r^{-2 / 3}\right)^{-1} .
$$

\section{Averaging rule for nanobulk}

The study of averaging rules for the effective properties of large polycrystals whose grains have anisotropic transport tensors extends back for nearly a century. ${ }^{17,18}$ Most work has focused on determining the theoretical upper and lower bounds of the effective conductivity, especially for configurations where the sample is macroscopically isotropic (obviously leading to $\mathbf{K}_{\text {eff }}=\kappa_{\text {eff }} \mathbf{I}$, where $\mathbf{I}$ is the identity tensor). The upper bound of $\kappa_{\text {eff }}$ for a bulk, macroscopically isotropic material is known to be identical to that given in Eq. (11), ${ }^{18,19}$ that is,

$$
\kappa_{\text {eff,bulk,UB }}=\kappa_{\text {eff,film }} .
$$

Similarly, the lower bound of $\kappa_{\text {eff }}$ is ${ }^{20}$

$$
\kappa_{\text {eff,bulk,LB }} / \kappa_{\text {char }}=\frac{1}{2} r^{2 / 3}\left(-1+\sqrt{1+8 r^{-1}}\right) .
$$

Both bounds have been proven to be realizable through carefully constructed hierarchical laminates. ${ }^{19,21}$

The practical self assembled structures of interest in this work $^{13}$ are considerably more random and less hierarchical than those proposed to realize $\kappa_{\text {eff,bulk,LB }}$ (Ref. 20) and $\kappa_{\text {eff,bulk,UB }},{ }^{19}$ so it is reasonable to expect that neither bound will be a good approximation for the $\kappa_{\text {eff,bulk }}$ of interest, especially when $r$ deviates significantly from unity. An approximate solution for the problem of a polycrystal with randomly oriented grains was obtained by Mityushov and Adamesku using a "correlational approximation" 22 which can be expressed as

$$
\kappa_{\text {eff,bulk,MA }} / \kappa_{\text {char }}=\frac{1}{3} r^{2 / 3}+\left[\frac{2}{3}-\frac{2}{9}\left(\frac{(r-1)^{2}}{r+2}\right)\right] r^{-1 / 3} .
$$

Although to our knowledge this result is unknown in the western literature, we shall see below that in practice Eq. (18) is significantly more accurate than either $\kappa_{\text {eff,bulk,LB }}$ or $\kappa_{\text {eff,bulk,UB }}$ at describing the effective thermal conductivity of randomized nanobulk materials.

\section{E. Numerical analysis using finite element methods (FEM)}

To verify the key results of Eqs. (11), (15), and (18), we performed a series of three-dimensional numerical simulations using FEM. As summarized in Table I, in all cases the "grains" were approximated as rectangular parallelepipeds of size $L_{x} \times L_{y} \times L_{z}$ stacked in a three-dimensional array measuring $N_{x} \times N_{y} \times N_{z}$ grains on each side. To best represent the conditions assumed in the derivations of Eqs. (11) and (15), the aspect ratios of those grains were chosen to be flat and elongated, respectively, while the grains for the bulk simulations were taken as cubes.

The boundary conditions chosen for each configuration are given in Table I. For lateral surfaces that represent the truncation of an infinite extent (e.g., the $x$ and $y$ faces of the film), we used periodic rather than adiabatic boundary conditions, because the latter would be equivalent to imposing an additional symmetry constraint beyond the symmetries already in the problem. By artificially restricting lateral heat flow, this would have impeded transport more than in a real crystal, resulting in a lower value of simulated thermal conductivity.

Using MATLAB to interface with COMSOL FEM software, every grain in every run was assigned a random thermal conductivity tensor. The tensors had prescribed principal 


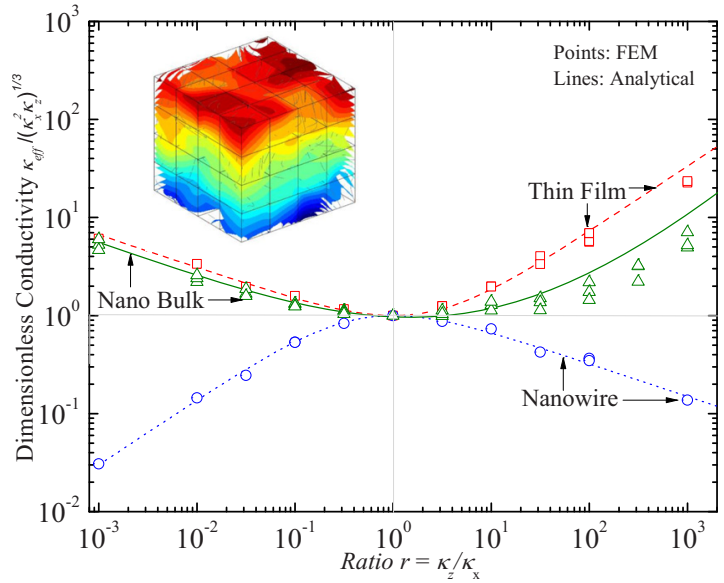

FIG. 2. (Color online) Averaging rules for the effective thermal conductivity of polycrystalline thin films (red squares), wires (blue circles), and nanobulk materials (green triangles). Points: FEM simulations of Table I. Lines: analytical results from Eqs. (11), (15), and (18). Inset: typical FEM simulation of a $4 \times 4 \times 4$ nanobulk configuration.

conductivities $\kappa_{x}=\kappa_{y}$ and $\kappa_{z}$. The thermal conductivity tensor of each grain was rotated into a random direction using $\mathbf{R}^{T} \mathbf{K R}$, where the rotation matrix $\mathbf{R}$ was randomized using an algorithm described in Ref. 23.

The results of these FEM calculations are summarized in Fig. 2, which spans six orders of magnitude in $r$. Although the scatter in the numerical results becomes significant at large $r$, Fig. 2 clearly confirms that Eqs. (11) and (15) are appropriate for films and wires, respectively. Figure 2 also reveals the clear difference between Eqs. (11) and (15): For a fixed value of $\kappa_{\text {char }}$, deviations of $r$ from unity result in increasing $\kappa_{\text {eff,film }}$ but decreasing $\kappa_{\text {eff,wire }}$, trends which are readily interpreted by visualizing these two systems as grains in parallel and in series, respectively.

For nanobulk materials, Fig. 2 also shows that the Mityushov-Adamesku (M-A) result of Eq. (18) is a good approximation to the FEM calculations for $r<10$, with agreement better than approximately $10 \%$ for $r<0.01$. For $r>100$, the numerical results are about half as large as the M-A approximation. We speculate that at least part of the disagreement is because these FEM calculations assumed cubic grains arrayed in a simple cubic lattice, because in this configuration each grain can only exchange heat with six neighboring grains. Unit cells with different symmetries can easily have a greater interconnectedness. For example, if the grains were arranged in a face-centered cubic (fcc) array, the shape of each grain would be a rhombic dodecahedron (the Wigner-Seitz cell of a fcc lattice ${ }^{24}$ ), which could exchange heat with 12 neighboring grains. A higher degree of interconnectedness would ease the lateral flow of heat around regions which by random chance had a grain aligned in such a way as to impede heat flow, and this should result in higher macroscopic thermal conductivity overall. This interconnectedness effect is expected to be more important in the limit of high rather than low $r$, because when $r \gg 1$, two out of the three principal directions will tend to block heat flow, and indeed, Fig. 2 shows that the disagreement between the FEM results and $\kappa_{\text {eff,bulk,MA }}$ is more significant for large rather than small $r$.
It is also evident from Fig. 2 that all of the geometries considered tend toward power law behaviors of $\kappa_{\text {eff }} / \kappa_{\text {char }}$ $\propto r^{2 / 3}$ or $\kappa_{\text {eff }} / \kappa_{\text {char }} \propto r^{-1 / 3}$ in the limits of large and/or small $r$. As explained in Appendix A, these power laws are easily understood from physical and dimensional considerations. Briefly, configurations dominated by the physics of parallel conductances (such as a thin film) must necessarily tend toward $\kappa_{\text {eff }} / \kappa_{\text {char }} \propto r^{2 / 3}$ for $r \gg 1$ and $\kappa_{\text {eff }} / \kappa_{\text {char }} \propto r^{-1 / 3}$ for $r \ll 1$; similarly, configurations dominated by the physics of resistances in series (e.g., a thin wire) must tend toward $r^{-1 / 3}(r$ $\gg 1)$ and $r^{2 / 3}(r \ll 1)$. For the nanobulk polycrystal, Fig. 2 and Eq. (18) clearly show that both the FEM and analytical calculations tend toward the same asymptotic power laws as the thin film. Thus, these power law trends show that the transport in the bulk polycrystal is best interpreted from the perspective of parallel conductances rather than series resistances.

\section{F. Sensitivity}

We define the dimensionless sensitivity $S_{\kappa_{x}}$ as the fractional change in $\kappa_{\text {eff }}$ for a unit fractional change in $\kappa_{x}$

$$
S_{\kappa_{x}}=\left.\frac{\kappa_{x}}{\kappa_{\text {eff }}} \frac{\partial \kappa_{\text {eff }}}{\partial \kappa_{x}}\right|_{\kappa_{z}}=\left.\frac{\partial\left[\ln \left(\kappa_{\mathrm{eff}}\right)\right]}{\partial\left[\ln \left(\kappa_{x}\right)\right]}\right|_{\kappa_{z}},
$$

and similarly for $S_{\kappa_{z}}$ upon exchanging $x \leftrightarrow z$. It is readily shown that the sensitivities obey a "sum rule;" $S_{\kappa_{x}}+S_{\kappa_{z}}=1$. The various averaging rules given above [e.g., Eqs. (11), (15), and (18)] are of the form $\kappa_{\mathrm{eff}, i} / \kappa_{\mathrm{char}}=f_{i}(r)$, where the index $i$ denotes the various configurations (film, wire, bulk, etc.), in which case

$$
S_{\kappa_{x}, i}=\frac{2}{3}-\frac{d\left[\ln \left(f_{i}\right)\right]}{d[\ln (r)]} .
$$

This expression is conveniently interpreted using the slopes of the log-log plot of Fig. 2. As an example, assuming randomly oriented grains, the thermal conductivity of a bulk polycrystal of graphite (which has $\kappa_{z} \ll \kappa_{x}$ ) will be fully sensitive to changes in $\kappa_{x}$, because $\{d[\ln (f)] / d[\ln (r)]\}_{r \ll 1}=$ $-1 / 3$ and thus $S_{\kappa_{x}} \approx 1$. At the same time, $\kappa_{\text {eff }}$ in a graphite polycrystal is completely insensitive to small changes in $\kappa_{z}$ $\left(S_{\kappa_{z}} \ll 1\right)$, thus quantifying the common notion that the heat flow in polycrystalline graphite is almost entirely along the basal planes within each grain. ${ }^{25,26}$

For the major $f_{i}(r)$ given above, explicit expressions for $S_{\kappa_{x}}$ are

$$
\begin{aligned}
& S_{\kappa_{x} \text { film }}=S_{\kappa_{x} \text { bulk,UB }}=\frac{2}{2+r}, \\
& S_{\kappa_{x}, \text { wire }}=\frac{2}{2+r^{-1}}, \\
& S_{\kappa_{x} \text {,bulk,MA }}=\frac{14 r^{2}+20 r+20}{(r+2)\left(r^{2}+16 r+10\right)} .
\end{aligned}
$$




\section{MODELING $\kappa_{x}$ AND $\kappa_{z}$ OF A SINGLE SUPERLATTICE GRAIN}

Following precedent, ${ }^{5,6,14}$ our approach is based on the BTE, which approximates the phonons as classical particles and neglects the possibility of coherent wave interference effects. This approximation is commonly applied to all but the shortest-period superlattices, and is particularly appropriate for the self-assembled materials of interest in this work because the random variations in the thicknesses of adjacent layers can be expected to be large in comparison to the typical phonon wavelengths (which are of the order of $1 \mathrm{~nm}$ at the Debye temperature and above ${ }^{27}$ ). A notable advance of the model described below is that the BTE solutions for both in-plane and cross-plane transport explicitly account for the frequency dependence of the phonon properties, whereas previous BTE solutions for cross-plane transport had been limited to the simpler "gray" or "effective phonon" approach. ${ }^{6,14}$

\section{A. Approximating the dispersion relations}

The phonon dispersion relations are approximated using an isotropic Born-von Karman/Einstein (BvKE) model for the acoustic and optical phonons, respectively, ${ }^{5,28}$

$$
\begin{aligned}
& \omega=\omega_{0} \sin \left(\frac{\pi q}{2 q_{0}}\right) \quad \text { (acoustic), } \\
& \omega=\omega_{E} \quad \text { (optical), }
\end{aligned}
$$

where $q$ is the wavevector, $q_{0}=\left(6 \pi^{2} \eta\right)^{1 / 3}$ is a cutoff wave vector, $\omega_{0}=2 v_{s} q_{0} / \pi$ is a characteristic frequency, $\eta$ is the number of primitive unit cells per unit volume, and $v_{s}$ is the sound velocity. The BvKE approach is nearly as convenient as a Debye approximation but is known to be significantly more accurate because of the more realistic treatment of the group velocities. ${ }^{28}$ On the other hand, compared to a full integration over the exact phonon dispersions, ${ }^{29}$ the BvKE approximation will be less accurate but is significantly more convenient, especially for adapting the model to materials whose full dispersion relations are not known, because a dispersion relation is fully specified by only three parameters $\left(\eta, v_{s}\right.$, and $\omega_{E}$, the last of which we assume does not affect steady-state heat transfer due to the low group velocity of optical phonons).

For a single polarization branch the density of states is

$$
\rho(\omega)=\frac{q^{2}}{2 \pi^{2} v},
$$

where $v=\partial \omega / \partial q$ is the group velocity. For simplicity we combine the one longitudinal and two transverse branches into one effective Born-von Karman (BvK) branch of threefold degeneracy, $v_{\mathrm{avg}}(\omega)$, so that the total density of states is

$$
\rho(\omega)=\frac{3 q^{2}}{2 \pi^{2} v_{\mathrm{avg}}} .
$$

Because here we are most interested in high temperatures $(T$ well above the Debye temperature $\theta_{D}$ ) and strong boundary scattering [all branches have approximately the same mean free path (MFP), related to the layer spacing], in this case it is easily shown that the most appropriate choice for the effective sound velocity is simply the arithmetic average of the three branches, $v_{s, \text { avg }}=(1 / 3) v_{s, L}+(2 / 3) v_{s, T}$. The volumetric specific heat per unit frequency $C(\omega)$ is

$$
C=\hbar \omega \rho \frac{\partial f}{\partial T},
$$

where $f$ is the Bose-Einstein distribution function.

\section{B. Phonon scattering mechanisms in bulk}

The BTE solutions below require as inputs the frequency-dependent MFPs of the bulk scattering mechanisms, $\Lambda_{\text {bulk }}(\omega)$. For common dielectrics and semiconductors, the two most important scattering mechanisms are generally impurity/defect scattering and phonon-phonon Umklapp scattering, the combined effect of which can be estimated using Matthiessen's rule, ${ }^{30}$

$$
\Lambda_{\text {bulk }}^{-1}=\Lambda_{\text {imp }}^{-1}+\Lambda_{\text {umkl }}^{-1} \text {. }
$$

Impurity/defect scattering is commonly approximated using a Rayleigh expression,

$$
\Lambda_{\mathrm{imp}}^{-1}=A_{1} \omega^{4} / v_{\mathrm{avg}},
$$

where $A_{1}$ is a fitting parameter that can be estimated from other properties. $^{31}$

For Umklapp scattering we use the common form ${ }^{32}$

$$
\Lambda_{\mathrm{umkl}}^{-1}=B_{1} \omega^{2} T \exp \left(-B_{2} / T\right) / v_{\mathrm{avg}}
$$

where $T$ is the absolute temperature and $B_{1}$ and $B_{2}$ are adjustable parameters. We note that $B_{2}$ can also be written $\theta_{D} /$ const, where const is generally not too different from $3 .{ }^{33}$ The numerical results presented below focus on $\mathrm{PbTe}\left(\theta_{D}\right.$ $\approx 136 \mathrm{~K})$ (Ref. 34) and $\operatorname{Sb}_{2} \mathrm{Te}_{3}\left(\theta_{D} \approx 160 \mathrm{~K}\right)($ Ref. 34) at room temperature and above. In this case $T \gg \theta_{D} / 3$, and furthermore as shown below we find that impurity scattering can be neglected, so Eq. (29) reduces to

$$
\Lambda_{\text {bulk }}^{-1}=\Lambda_{\mathrm{umkl}}^{-1}=B_{1} \omega^{2} T / v_{\mathrm{avg}} .
$$

Thus, only one scattering parameter $\left(B_{1}\right)$ for each material is needed to complete the model for the phonon thermal conductivity.

To assess the validity of the assumptions leading to Eq. (32), we compare the theoretical results obtained using this assumption with experimental data from the literature for the phonon thermal conductivity of PbTe (Refs. 35 and 36) and $\mathrm{Bi}_{2} \mathrm{Te}_{3} \cdot{ }^{37}$ As shown in Fig. 3, the agreement is very good above $100 \mathrm{~K}$. In particular we note that the data very closely follow a $T^{-1}$ trend over the temperatures of interest, as expected due to high-temperature Umklapp scattering [verifying Eq. (32)] combined with a nearly constant specific heat (law of DuLong and Petit: $T>\theta_{D}$ ). This implies that related thermoelectric materials can be modeled by using only a single experimental thermal conductivity data point to fix $B_{1}$. This is an essential simplification for our modeling of $\mathrm{Sb}_{2} \mathrm{Te}_{3}$, for which we were unable to locate temperaturedependent thermal conductivity measurements from the literature. ${ }^{38,39}$ Because of the close similarities in the crystal 


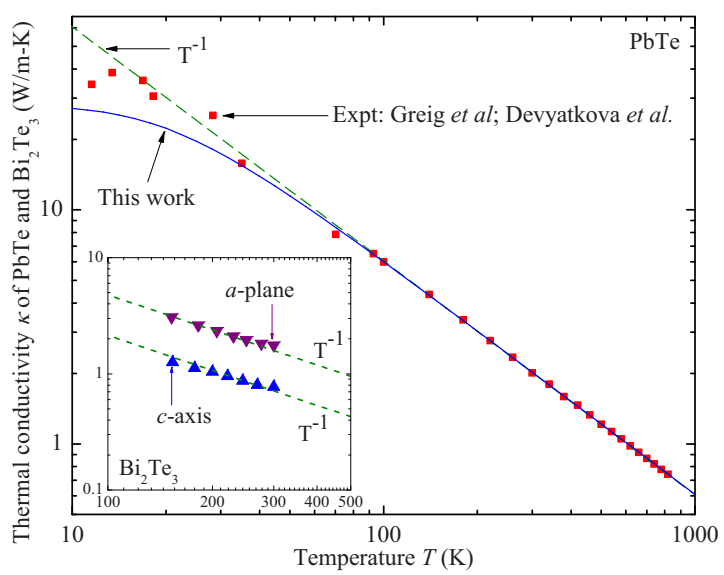

FIG. 3. (Color online) Theoretical thermal conductivity of PbTe using only the high-temperature Umklapp expression of Eq. (32) (blue solid line), as compared to experimental data from Greig in Ref. 35 and Devyatkova in Ref. 36 (red squares). Above $80 \mathrm{~K}$, the data and model follow a $T^{-1}$ relation almost perfectly (dashed green line). Inset: experimental data (Ref. 37) (points) for the thermal conductivity of $\mathrm{Bi}_{2} \mathrm{Te}_{3}$ in both $c$-axis and $a$-plane directions also follows a $T^{-1}$ relation around room temperature.

structures of $\mathrm{Sb}_{2} \mathrm{Te}_{3}$ and $\mathrm{Bi}_{2} \mathrm{Te}_{3}$, and considering the success of Eq. (32) at modeling $\mathrm{Bi}_{2} \mathrm{Te}_{3}$ in Fig. 3, we apply Eq. (32) to $\mathrm{Sb}_{2} \mathrm{Te}_{3}$ based only on the room-temperature results from Ref. 39. The resulting values of $B_{1}$ for the $a$-plane and $c$-axis directions are given in Table II, along with other property values used in this work.

\section{Considerations for anisotropic constituent materials}

The BTE solutions described below are only strictly appropriate for superlattices made up of isotropic materials. This is a good approximation for cubic materials such as $\mathrm{Si}$, $\mathrm{Ge}, \mathrm{GaAs}$, and $\mathrm{PbTe}$, because their dispersion relations are approximately isotropic and their bulk transport properties are exactly so. However, $\mathrm{Sb}_{2} \mathrm{Te}_{3}$ is a hexagonal material which exhibits significant anisotropy between the $a$-plane and $c$-axis directions. Developing an exact BTE theory for strongly anisotropic materials is beyond the scope of this work, so we make several approximations (Table II). We assume that the $c$-axis of the $\mathrm{Sb}_{2} \mathrm{Te}_{3}$ layers is always aligned perpendicular to the superlattice planes. When calculating

TABLE II. Properties for $\mathrm{PbTe}$ and $\mathrm{Sb}_{2} \mathrm{Te}_{3}$ at $300 \mathrm{~K}$. The adjustable parameters $B_{1}$ for $\mathrm{Sb}_{2} \mathrm{Te}_{3}$ were fitted using the room temperature bulk phonon thermal conductivities $\kappa_{\mathrm{ph}, \mathrm{FL}}$ in Ref. 39. Densities of primitive unit cells $\eta$ are calculated using the lattice constant and crystal structure. The sound velocities $v_{s}$ of $\mathrm{Sb}_{2} \mathrm{Te}_{3}$ are estimated from those of $\mathrm{Bi}_{2} \mathrm{Te}_{3}$ using the scaling arguments explained in Appendix B.

\begin{tabular}{lccc}
\hline \hline Parameters & $\mathrm{PbTe}$ & $\mathrm{Sb}_{2} \mathrm{Te}_{3}(a$-plane $)$ & $\mathrm{Sb}_{2} \mathrm{Te}_{3}(c$-axis $)$ \\
\hline$B_{1}\left(10^{-18} \mathrm{~s} / \mathrm{K}\right)$ & $6.2^{\mathrm{a}}$ & 4.3 & 27.8 \\
Lattice constant $(\AA)$ & $6.462^{\mathrm{b}}$ & $4.25^{\mathrm{b}}$ & $30.35^{\mathrm{b}}$ \\
$\eta\left(10^{28} \mathrm{~m}^{-3}\right)$ & 1.482 & 0.6329 & 0.6329 \\
$v_{s}(\mathrm{~m} / \mathrm{s})$ & $1730^{\mathrm{a}}$ & 2333 & 2270 \\
$\kappa_{\mathrm{ph}, \mathrm{FL}}$ at $300 \mathrm{~K}(\mathrm{~W} / \mathrm{m} \mathrm{K})$ & $2.0^{\mathrm{a}}$ & $2.2^{\mathrm{c}}$ & $0.34^{\mathrm{c}}$ \\
\hline
\end{tabular}

${ }^{\mathrm{a}}$ Reference 27.

${ }^{\mathrm{b}}$ Reference 34.

${ }^{\mathrm{c}}$ Reference 39. the BTE solutions in the cross-plane direction of the superlattice, we take the effective $\mathrm{Sb}_{2} \mathrm{Te}_{3}$ sound velocity to be the $c$-axis value and determine the $\mathrm{Sb}_{2} \mathrm{Te}_{3}$ Umklapp parameter $B_{1}$ from the bulk experimental thermal conductivity in the $c$-axis direction. Similarly, for the BTE superlattice solutions in the in-plane direction, we determine the $\mathrm{Sb}_{2} \mathrm{Te}_{3} v_{s}$ and $B_{1}$ from the $a$-axis literature values.

\section{In-plane thermal conductivity $\kappa_{x}$}

To calculate the thermal conductivity along the superlattice planes we use directly the analytical BTE solution given by Chen, ${ }^{5}$

$$
\begin{aligned}
\kappa_{x}= & \sum_{i=1}^{2} \frac{d_{i}}{d_{1}+d_{2}} \int_{0}^{\omega_{0, m}} \frac{1}{3} C_{i} v_{\text {avg }, i} \Lambda_{\text {bulk }, i} \\
& \times\left\{1-\frac{3 \Lambda_{\text {bulk }, i}}{2 d_{i}}\left[p G_{s i}\left(\xi_{1}, \xi_{2}\right)+(1-p) G_{d i}\left(\xi_{1}, \xi_{2}\right)\right]\right\} d \omega,
\end{aligned}
$$

where $i=1,2$ represents the two different layers, $d_{i}$ is the corresponding layer thickness, $\omega_{0, m}$ is the frequency cutoff, $\xi_{i}=d_{i} / \Lambda_{\mathrm{bulk}, i}, G_{s i}$ and $G_{d i}$ are dimensionless integral functions given in Ref. 5, and $p$ is the specularity parameter which captures the effects of the interfacial roughness. A perfectly smooth interface corresponds to $p=1$ (specular transmission/reflection), while a very rough interface corresponds to $p=0$ (diffuse). For surfaces of intermediate roughness $p$ can be estimated using $p=\exp \left(-16 \pi^{2} \delta^{2} / \lambda^{2}\right)$, where $\delta$ is the root mean square surface roughness and $\lambda$ is the phonon wavelength, and we have confirmed Zhang's ${ }^{40}$ observation that the expression given in Ziman's book ${ }^{41}$ contained an erroneous factor of $\pi$.

\section{E. Cross plane thermal conductivity $\kappa_{z}$}

Significant reductions in the cross plane thermal conductivity $\kappa_{z}$ in superlattices have been observed in experiments ${ }^{2,42}$ and investigated by solving the BTE. ${ }^{6,14}$ For example, Chen ${ }^{6}$ considered several different mechanisms of phonon scattering at an interface, such as elastic, inelastic, diffusive, and specular scattering. He found that the inelastic acoustic mismatch model (AMM) has better agreement with experiment than the elastic AMM. To simplify the calculation, Chen used averaging procedures to approximate all phonon properties as frequency-independent (also known as a "gray media" approximation). However, from previous work on nanowires it is known that the gray-media approach will overpredict the thermal conductivity of a nanostructure, and that better agreement with experiments is achieved by using BTE solutions that account for the full frequency dependence of the phonon group velocity and MFPs. ${ }^{43}$ Therefore, here we extend the gray-medium model of Ref. 6 to account for the phonon frequency-dependence without averaging.

In our frequency-dependent model we break the BvK dispersion relation of Eq. (33) into $l$ very small frequency bands. In the $i$-th layer, where $i=1,2$ still represents the two different layers, the $j$-th band centers are denoted $\omega_{i j}$, with 
frequency spread $\Delta \omega_{i j}$ and wave vector spread $\Delta q_{i j}$ $=\Delta \omega_{i j} / v\left(\omega_{i j}\right)$. Around each $\omega_{i j}$, the dispersion relation is approximately linear and we can approximate the specific heat, group velocity, and MFP as being independent of frequency. Thus, for each small band the gray media model can still be applied. Thus, the volumetric specific heat for a single band in the $i$-th layer and $j$-th frequency band is

$$
\Delta C_{i j}=\hbar \omega_{i j} \rho\left(\omega_{i j}\right) \Delta \omega_{i j} \frac{\partial f\left(\omega_{i j}, T\right)}{\partial T} .
$$

In each small frequency band the bulk MFP is still given by Eq. (32), and the gray-BTE model of Ref. 6 ,

$$
\sin \theta_{i j} \cos \varphi_{i j} \frac{\partial I_{i j}}{\partial x}+\cos \theta_{i j} \frac{\partial I_{i j}}{\partial z_{i}}=-\frac{I_{i j}-I_{o i j}}{\Lambda_{i j}},
$$

can be solved numerically. Here $\theta_{i j}$ and $\phi_{i j}$ are the polar and azimuthal angles of local grain system as shown in Fig. 1(b), and $I_{i j}$ and $I_{o i j}$ indicate the phonon intensity and the equilibrium phonon intensity in the $i$-th layer and the $j$-th frequency band. Thus, using the methods of Ref. 6, we can obtain the cross-plane thermal conductivity $\Delta \kappa_{z j}$ for this band. Finally, the total cross plane thermal conductivity is found by summing

$$
\kappa_{z}=\sum_{j=1}^{l} \Delta \kappa_{z j} .
$$

For each superlattice layer the numerical solution of Eq. (35) requires a spatial integration over $z$ and an angular integration over $\theta$. Because the temperature profiles are more sharply varying near the interfaces, for the spatial integration we follow Ref. 6 and use a Gauss-Legendre method because it assigns the mesh points with finer spacings near the two limits of integration. The angular integration faces an additional challenge because of the need to match the mesh of incident and transmitted angles on each side of an interface according to inelastic $\mathrm{AMM},{ }^{6}$

$$
\frac{\sin \theta_{1 j}}{\sin \theta_{2 j}}=\left(\frac{\Delta C_{2 j} v_{2 j}}{\Delta C_{1 j} v_{1 j}}\right)^{1 / 2} \text {. }
$$

To satisfy this constraint we implement a trapezoidal integration scheme. Following Ref. 14, we mesh the polar angle $\theta_{2 j}$ of the $\mathrm{Sb}_{2} \mathrm{Te}_{3}$ layer uniformly into $m$ equal parts, and then use Eq. (37) to determine the corresponding (nonuniform) angular mesh of $\theta_{1 j}$ in the adjacent $\mathrm{PbTe}$ layer.

\section{NUMERICAL RESULTS AND DISCUSSION}

We now present numerical results for the effective thermal conductivity $\kappa_{\text {eff }}$ of a bulk polycrystal made of randomly oriented superlattice grains of $\mathrm{PbTe} / \mathrm{Sb}_{2} \mathrm{Te}_{3}$, including the effects of period, specularity, temperature, and the difference between gray and frequency-dependent modeling for $\kappa_{z}$. The calculations extend to periods $L$ down to $1 \mathrm{~nm}$, although we note that the assumption of classical phonon particles is expected to break down in the single-nanometer regime where coherent wave effects may become important. ${ }^{3,4}$ In all cases we fix the layer thicknesses in the ratio $d_{1}=d_{\mathrm{PbTe}}=2 L / 9$ and $d_{2}=d_{\mathrm{Sb}_{2} \mathrm{Te}_{3}}=7 L / 9$, consistent with Ref. 13 .
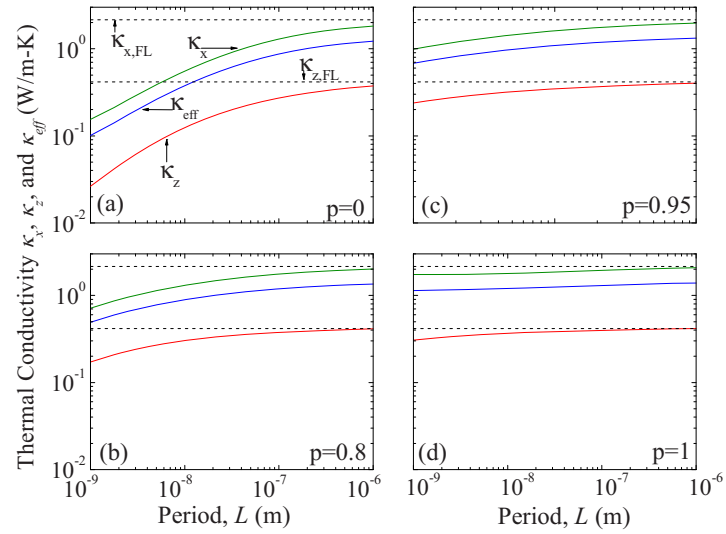

FIG. 4. (Color online) Thermal conductivity as a function of period for four different values of the specularity parameter $p$, for a $\mathrm{PbTe}-\mathrm{Sb}_{2} \mathrm{Te}_{3}$ nanobulk system at $T=300 \mathrm{~K}$ with thickness ratio $\mathrm{PbTe}: \mathrm{Sb}_{2} \mathrm{Te}_{3}=2 / 9: 7 / 9$. Solid lines: $\kappa_{x}$ and $\kappa_{z}$ are the in-plane and cross-plane values for a single superlattice grain, while $\kappa_{\text {eff }}$ is the value for a bulk polycrystal with randomly oriented grains. Dashed lines: $\kappa_{x, \mathrm{FL}}$ and $\kappa_{z, \mathrm{FL}}$ are the classical Fourier-law values for a single superlattice grain, neglecting phonon size effects.

\section{A. Effect of period}

Figure 4 shows the thermal conductivities as a function of period for four different specularities. In each panel, the three solid lines correspond to the in-plane (green, highest curve) and cross-plane (red, lowest curve) thermal conductivities of a single superlattice grain, as well as $\kappa_{\text {eff }}$ of a bulk polycrystal (blue, intermediate curve) obtained using the $\mathrm{M}-\mathrm{A}$ averaging rule of Eq. (18). In all cases, at large periods the calculations approach the correct classical Fourier Law limits (dashed lines) for in-plane

$$
\kappa_{x, \mathrm{FL}}=\frac{\left(d_{1} \kappa_{1, \mathrm{FL}}+d_{2} \kappa_{2, \mathrm{FL}}\right)}{d_{1}+d_{2}},
$$

and cross-plane

$$
\kappa_{z, \mathrm{FL}}^{-1}=\frac{\left(d_{1} \kappa_{1, \mathrm{FL}}^{-1}+d_{2} \kappa_{2, \mathrm{FL}}^{-1}\right)}{d_{1}+d_{2}},
$$

where $\kappa_{1, \mathrm{FL}}$ and $\kappa_{2, \mathrm{FL}}$ are the bulk thermal conductivities for layers 1 and 2 .

Figure 4 also shows that the increased phonon scattering at small periods affects in-plane and cross-plane transport approximately equally, because the ratio $r=\kappa_{z} / \kappa_{x}$ remains nearly constant around $0.17-0.25$ in all cases. Recall from Sec. II E that the heat transfer in a bulk polycrystal is best understood as dominated by the physics of thermal conductances in parallel, that is, the heat will tend to locally flow along "the leakiest path" within each grain: whichever direction (in-plane or cross-plane) has the largest principal thermal conductivity ( $\kappa_{x}$ or $\kappa_{z}$, respectively). In terms of sensitivity [Eq. (23)], for $r<0.25$ here we find $S_{\kappa_{x}}>0.82$ (and thus $\left.S_{\kappa_{z}}<0.18\right)$. Thus, a $10 \%$ reduction in $\kappa_{x}$ will reduce $\kappa_{\text {eff }}$ by $8.2 \%$, while a $10 \%$ reduction in $\kappa_{z}$ would only reduce $\kappa_{\text {eff }}$ by $1.8 \%$. This observation shows that efforts to manipulate the thermal conductivity of the bulk polycrystal should focus primarily on $\kappa_{x}$ rather than $\kappa_{z}$. We also note that Fig. 4 and the classical limits of Eqs. (38) and (39) suggest that $\kappa_{z}$ $<\kappa_{x}$ is always expected, although in this $\mathrm{PbTe} / \mathrm{Sb}_{2} \mathrm{Te}_{3}$ sys- 


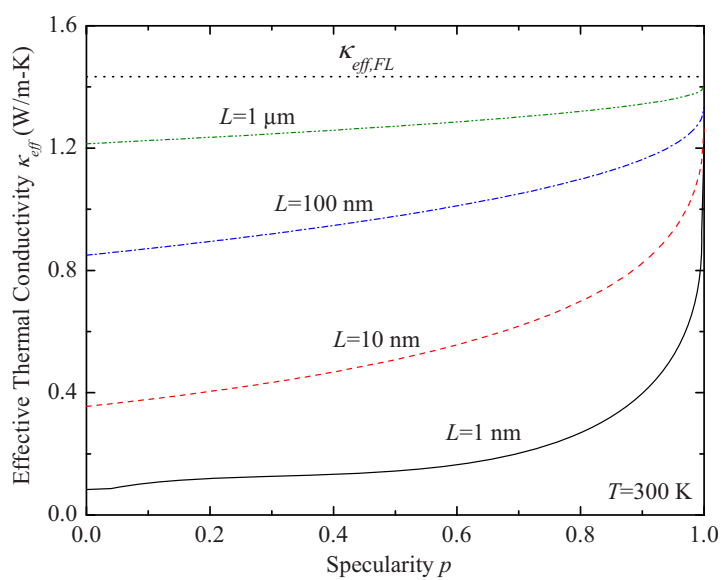

FIG. 5. (Color online) Bulk effective thermal conductivity as a function of specularity for four different periods $L$, for a $\mathrm{PbTe}-\mathrm{Sb}_{2} \mathrm{Te}_{3}$ nanobulk system at $T=300 \mathrm{~K}$ with thickness ratio $\mathrm{PbTe}: \mathrm{Sb}_{2} \mathrm{Te}_{3}=2 / 9: 7 / 9$.

tem the effect is even stronger because of our assumption that the $\mathrm{Sb}_{2} \mathrm{Te}_{3} c$-axis (its low $\kappa$ direction) is aligned in the $z$ direction of the superlattice.

\section{B. Effect of specularity}

Figure 5 shows $\kappa_{\text {eff }}$ for a bulk $\mathrm{PbTe}-\mathrm{Sb}_{2} \mathrm{Te}_{3}$ polycrystal as a function of specularity for four different periods. For comparison, the value of $\kappa_{\text {eff,FL }}$ is found from Eqs. (18), (38), and (39). It is clear that diffuse scattering (smaller $p$ ) always tends to reduce the thermal conductivity, because in these systems diffuse scattering is always more effective than specular scattering at impeding phonon transport. In all cases $\kappa_{\text {eff }}$ is most sensitive to changes in $p$ for large $p$, especially for the smaller periods because of the greater density of interfaces. For example, for $L=10 \mathrm{~nm}, 62 \%$ of the transition in $\kappa_{\text {eff }}$ occurs over the relatively narrow range of $1>p$ $>0.8$, while the remaining $38 \%$ of the transition in $\kappa_{\text {eff }}$ is distributed over the large range $0.8>p>0$.

We also note from Figs. 4 and 5 that $p=1$ is not necessarily sufficient to recover the Fourier Limit values of $\kappa_{x, \mathrm{FL}}$ and $\kappa_{z, \mathrm{FL}}$. Rather, it is also necessary that the layer thicknesses should be significantly larger than the bulk MFPs. This effect has been reported previously for BTE solutions in both the in-plane ${ }^{5}$ and cross-plane ${ }^{6}$ directions.

\section{Effect of temperature}

Figure 6 shows the temperature dependence of the effective thermal conductivity for a fixed period of $10 \mathrm{~nm}$. For perfectly specular interfaces $(p=1), \kappa_{\text {eff }}$ decreases from 1.21 $\mathrm{W} / \mathrm{m} \mathrm{K}$ at $300 \mathrm{~K}$ to $0.73 \mathrm{~W} / \mathrm{m} \mathrm{K}$ at $500 \mathrm{~K}$, a reduction by $40 \%$ which approximately follows a $T^{-1}$ trend which is slightly below the bulk classical value. However, in the other limit of perfectly rough interfaces $(p=0)$, the reduction in $\kappa_{\text {eff }}$ is weaker, from 0.378 to $0.288 \mathrm{~W} / \mathrm{m} \mathrm{K}$ over this same temperature range, or only a $24 \%$ reduction which can be approximated by a weaker $T^{-0.52}$ power law. The different behavior is due to the different scattering mechanisms that dominate these two cases. For the perfectly specular case, the interfacial scattering is relatively weak as compared to the Umklapp scattering, which over this temperature range fol-

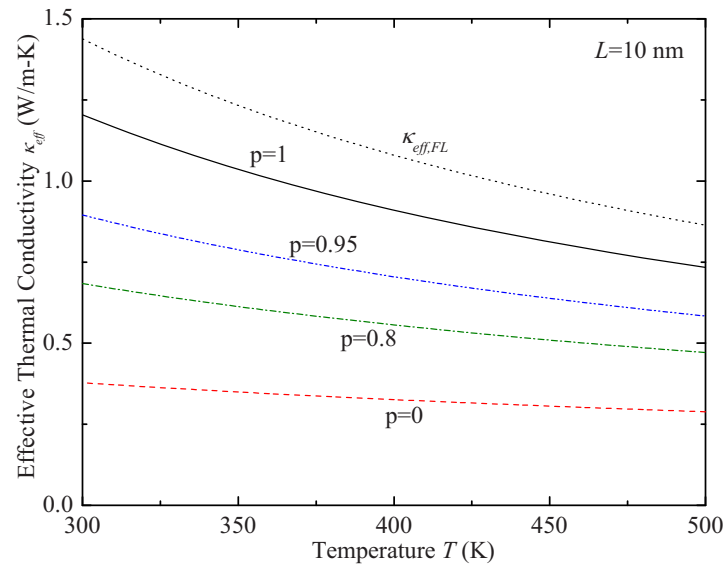

FIG. 6. (Color online) Bulk effective thermal conductivity as a function of temperature for four different values of the specularity $p$, for a $\mathrm{PbTe}-\mathrm{Sb}_{2} \mathrm{Te}_{3}$ system with thickness ratio $\mathrm{PbTe}: \mathrm{Sb}_{2} \mathrm{Te}_{3}=2 / 9: 7 / 9$ and fixed period $L=10 \mathrm{~nm}$. The black dotted line is the bulk classical Fourier law value.

lows a $T^{-1}$ trend for both bulk PbTe and bulk $\mathrm{Sb}_{2} \mathrm{Te}_{3}$ (recall Fig. 3). However, in the perfectly diffuse case, the interfacial scattering makes a significant contribution to reducing the total thermal conductivity. Because the effective MFP of boundary scattering is nearly independent of temperature, increasing boundary scattering tends to weaken the $T^{-1}$ trend of the Umklapp scattering, which finally gives the observed $T^{-0.52}$ behavior.

\section{Comparison of gray versus frequency dependent modeling}

Recall from Sec. III E that previous ${ }^{6,14}$ BTE solutions for $\kappa_{z}$ had relied on the gray media approximation, while here we have developed a frequency-dependent solution. These two approaches are compared in Fig. 7. In the limit $L \rightarrow \infty$, boundary scattering is negligible as compared to Umklapp scattering, and both models converge to the same bulk value, independent of specularity. In the opposite limit of $L \rightarrow 0$, the boundary scattering is much more important than Umklapp

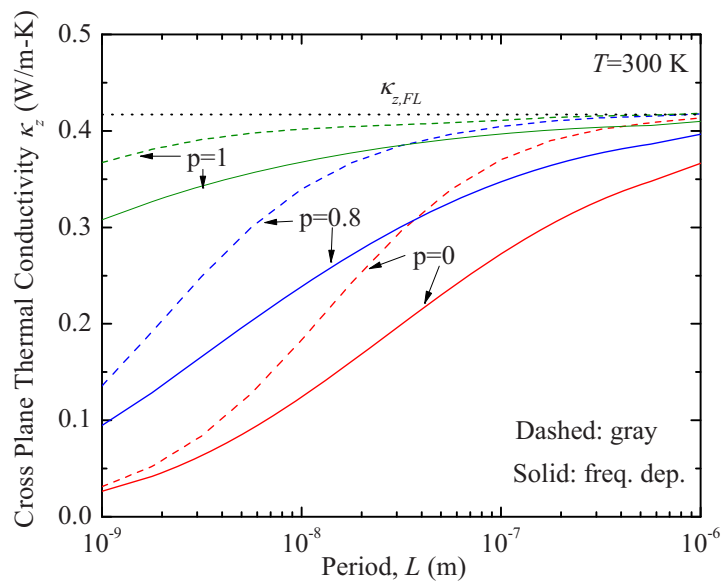

FIG. 7. (Color online) Comparison of present frequency-dependent model (solid lines) and traditional gray media model (dashed lines) for the perioddependence of the cross-plane superlattice thermal conductivity $\kappa_{z}$. The calculations are for a $\mathrm{PbTe}-\mathrm{Sb}_{2} \mathrm{Te}_{3}$ superlattice system at $T=300 \mathrm{~K}$ with thickness ratio $\mathrm{PbTe}: \mathrm{Sb}_{2} \mathrm{Te}_{3}=2 / 9: 7 / 9$. 
TABLE III. Fraction transformed $(Y)$ and interlamellar spacing of the samples used for lattice thermal conductivity measurements.

\begin{tabular}{|c|c|c|c|c|c|}
\hline \multirow[b]{2}{*}{ Sample ID } & \multicolumn{2}{|c|}{ Annealing condition } & \multirow{2}{*}{$\begin{array}{r}Y \\
(\%)\end{array}$} & \multicolumn{2}{|c|}{$\begin{array}{c}\text { Period } \\
(\mathrm{nm})\end{array}$} \\
\hline & $T / \mathrm{K}$ & $t / \mathrm{h}$ & & Average & Standard deviation \\
\hline 1 & 573 & 840 & 100 & 283 & 76 \\
\hline \multirow[t]{2}{*}{2} & 673 & 78 & 99.7 & 536 & 155 \\
\hline & & 150 & 100 & 575 & 153 \\
\hline \multirow[t]{2}{*}{3} & 773 & 1 & 88.7 & 544 & 168 \\
\hline & & 126 & 100 & 1591 & 487 \\
\hline
\end{tabular}

scattering, and again the frequency-dependent and gray media models converge to the same values (for a fixed value of $p)$. This convergence is most clear for the $p=0$ curves in Fig. 7 , while the convergence for the $p=0.8$ and $p=1$ curves is only evident for periods well below $1 \mathrm{~nm}$, which are unphysical because this framework neglects the granularity of the lattice.

The most important feature of Fig. 7 is in the transition regimes of moderate periods, where the gray media model fails to capture the full reduction in thermal conductivity that is seen with the frequency-dependent model. For example, at $L=200 \mathrm{~nm}$ and $p=0$, the thermal conductivity reduction as compared to bulk is only $6.6 \%$ for the gray media model, but $27 \%$ for the frequency-dependent model. We also note that the transition between small-period and large-period behaviors spans a larger range of $L$ in the frequency-dependent model. Similar observations have been described in models of silicon nanowires, ${ }^{43}$ and can be understood in terms of the distributions of phonon mean paths that contribute to the total thermal conductivity. ${ }^{27}$ Gray media models lump all Umklapp scattering [Eq. (31)] together into a single value of the effective MFP, $\Lambda_{\text {lump }}$. Clearly, for $L \gg \Lambda_{\text {lump }}$ the bulk value of $\kappa_{z, \mathrm{FL}}$ is recovered, and for $L \ll \Lambda_{\text {lump }}$ the boundaryscattering limit is recovered. However, by accounting for the frequency dependence of the Umklapp MFPs, the present model effectively "smears out" the importance of the MFPs over a broad range, typically spanning more than two orders of magnitude in $\Lambda .{ }^{27}$ Thus, in a frequency-dependent model the long- $\Lambda$ portion of the distribution begins to experience significant boundary scattering at much larger periods than the equivalent gray-media model, explaining the separation between dashed and solid lines in Fig. 7 at moderate $L$.

\section{COMPARISON WITH EXPERIMENT}

Samples with overall composition matching the " $\mathrm{Pb}_{2} \mathrm{Sb}_{6} \mathrm{Te}_{11}$ " compound $\left(\mathrm{Pb}_{10.5} \mathrm{Sb}_{31.6} \mathrm{Te}_{57.9}\right)$ and weighing 15-20 g were prepared by injection molding using a copper mold with $20 \times 30 \times 3 \mathrm{~mm}^{3}$ dimension. Details for the injection molding are given in Ref. 44. Plate samples with 10 $\times 10 \mathrm{~mm}^{2}$ size were cut out with a diamond saw and then the surfaces were ground to remove the surface layers until the final thickness was around $1.5 \mathrm{~mm}$. Electrical resistivity and thermal conductivity were measured before and after annealing. For annealing, samples were sealed under vacuum in fused silica tubes. The annealing temperature was $573 \mathrm{~K}$ $(840$ h), $673 \mathrm{~K}(78 \mathrm{~h}, 150$ h), or $773 \mathrm{~K}(1 \mathrm{~h}, 126 \mathrm{~h})$.
The microstructures were observed using a field emission-scanning electron microscope (Carl Zeiss LEO $1550 \mathrm{VP})$ equipped with a backscattered electron detector for its high compositional contrast capabilities. The microstructures were found to be essentially the same as those reported previously. ${ }^{13}$ The microstructures were digitally analyzed using an image analysis program (Macscope, Mitani Corp.) to determine the interlamellar spacing (period) and the fraction transformed $(Y)$. The method to determine the true period is given in Ref. 45. The results are summarized in Table III. The temperature and time dependences of the period have been discussed previously. ${ }^{13,46}$

The electrical resistivity $(\rho)$ as a function of temperature was measured using the van der Pauw method with a current of $10 \mathrm{~mA}$. The Hall coefficient $\left(R_{\mathrm{H}}\right)$ was measured in the same apparatus with a forward and reverse magnetic field value of $\sim 9500 \mathrm{G}$. The carrier density $(n)$ was calculated from the Hall coefficient assuming a scattering factor of 1.0 in a single-carrier scheme, with $n=1 / R_{\mathrm{H}} e$, where $n$ is the density of charge carriers (holes) and $e$ the charge of the electron. The thermal diffusivities were measured at $300 \mathrm{~K}$ by flash diffusivity technique (LFA457, NETSZCH). The thermal conductivities $\left(\kappa_{\text {tot }}\right)$ were calculated from the measured thermal diffusivity $(\alpha)$, the measured density $(\rho)$, and the heat capacity $\left(C_{\mathrm{P}}\right)$ evaluated by Dulong-Petit law using the relation, $\kappa_{\mathrm{tot}}=\rho C_{\mathrm{P}} \alpha$. The electron part of the thermal conductivity, $\kappa_{\mathrm{el}}$ was evaluated using the Wiedemann-Franz law, $\kappa_{\mathrm{el}}=L T / \rho$, where $L$ is the Lorenz number, 2.45 $\times 10^{-8} \Omega \mathrm{K}^{-2}$. The phonon part of the thermal conductivity, $\kappa_{\mathrm{ph}}$, was calculated using $\kappa_{\mathrm{tot}}=\kappa_{\mathrm{el}}+\kappa_{\mathrm{ph}}$ and is plotted as a function of period in Fig. 8. [Some data points have been reported in Ref. 47. In the present paper, the points at $283 \mathrm{~nm}$ period for $\mathrm{PbTe}-\mathrm{Sb}_{2} \mathrm{Te}_{3}$ lamellae (annealed at $573 \mathrm{~K}$ ) and at $1.4 \mathrm{~nm}$ for $\mathrm{Pb}_{2} \mathrm{Sb}_{6} \mathrm{Te}_{11}$ (before annealing) were added.] In the figure, the data points for $\mathrm{Pb}_{2} \mathrm{Sb}_{6} \mathrm{Te}_{11}$ obtained before annealing are also plotted using an equivalent period of 1.4 $\mathrm{nm}$, obtained from the size of a single atomic unit cell of $\mathrm{Pb}_{2} \mathrm{Sb}_{6} \mathrm{Te}_{11}$ (Ref. 48).

\section{A. Comparison between model and experiment}

Using the parameters of Table II, the highest effective thermal conductivity seen in our calculations is the Fourier limit $\kappa_{\text {eff,FL }}=\kappa_{\text {eff }}(L \rightarrow \infty)=1.43 \mathrm{~W} / \mathrm{m} \mathrm{K}$. However, the experimental results above imply phonon thermal conductivities that are much larger than this; for example, for $L$ $=1590 \mathrm{~nm}$, the experimental phonon $\kappa_{\text {eff }}$ is estimated as 3.87 


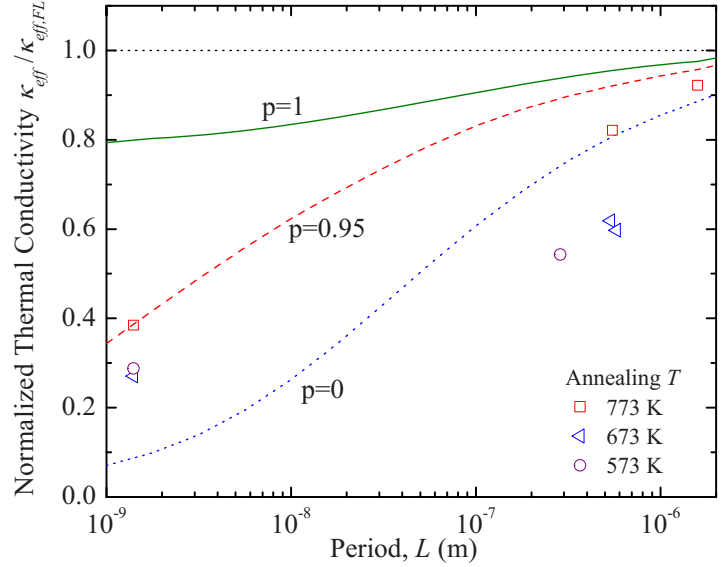

FIG. 8. (Color online) Comparison of normalized theoretical (lines) and measured (points) thermal conductivity at $T=300 \mathrm{~K}$. The theoretical results are normalized as $\kappa_{\mathrm{eff}}(L) / 1.43 \mathrm{~W} / \mathrm{m} \mathrm{K}$ and the experimental results as $\kappa_{\text {eff }}(L) / 4.21 \mathrm{~W} / \mathrm{m} \mathrm{K}$.

$\mathrm{W} / \mathrm{m} \mathrm{K}$, which exceeds the model $\kappa_{\text {eff,FL }}$ as well as all of the bulk values assumed in Table II. We attribute this disagreement between model and experiment to two major effects: (1) from the modeling side, it is difficult to determine accurate values for the phonon thermal conductivity of bulk $\mathrm{Sb}_{2} \mathrm{Te}_{3}$. The values in Table II are taken from Ref. 39, which itself is the result of a theoretical analysis rather than a direct experimental measurement. (2) From the experimental side, in subtracting the $\kappa_{\mathrm{el}}$ from $\kappa_{\mathrm{tot}}$ we assumed that a macroscopically-averaged Wiedemann-Franz law can be applied to the effective properties of the bulk polycrystal, and that the Lorenz number was known. The Lorenz number used is valid for degenerate (metallic) semiconductors. The low thermopower observed in these samples $(30 \mu \mathrm{V} / \mathrm{K})$ (Ref. 13) is indicative of degenerate behavior despite the relatively low carrier concentration observed by Hall effect. ${ }^{47}$ The ambipolar effect ${ }^{11,49}$ is also expected to be significant in these samples, whereby the formation and recombination of electron-hole pairs contributes to $\kappa$ but not $\sigma$. Thus, the experimental quantity identified as $\kappa_{\mathrm{ph}}$ is actually $\kappa_{\text {ph }}+\kappa_{\text {Ambipolar }}$, making it an overestimate of the true phonon contribution.

Because of the difficulties in reconciling the model and experimental values directly, we instead focus on a comparison of normalized quantities. First, to study the effect of the period, Fig. 8 shows the thermal conductivities normalized to their bulk values, that is, $\kappa_{\text {eff }}(L) / \kappa_{\text {eff }}(L \rightarrow \infty)$. For the model calculation, $\kappa_{\text {eff }}(L \rightarrow \infty)=\kappa_{\text {eff, FL }}=1.43 \mathrm{~W} / \mathrm{m} \mathrm{K}$. For the experiments, the largest period available is $L=1590 \mathrm{~nm}$, which may not necessarily have recovered to its $L \rightarrow \infty$ value. From the theoretical calculations, the ratio $\kappa_{\text {eff }}(1590) / \kappa_{\text {eff }}(\infty)$ ranges from $88.6 \%(p=0)$ to $95.8 \%(p=0.95)$. We take the average of these two $(92.2 \%)$ as an estimate for the experimental $\kappa_{\text {eff }}(1590) / \kappa_{\text {eff }}(\infty)$, to finally estimate $\kappa_{\text {eff }}(\infty)$ $\approx 4.21 \mathrm{~W} / \mathrm{m} \mathrm{K}$ for the experiments.

Figure 8 also shows data for the as-quenched metastable $\mathrm{Pb}_{2} \mathrm{Te}_{6} \mathrm{Sb}_{11}$ phase, which was assigned a "period" of $L$ $=1.4 \mathrm{~nm}$ based on the crystal structure. Although it appears that the particle-based BTE theory of this work may also give a good estimate of the thermal conductivity of the

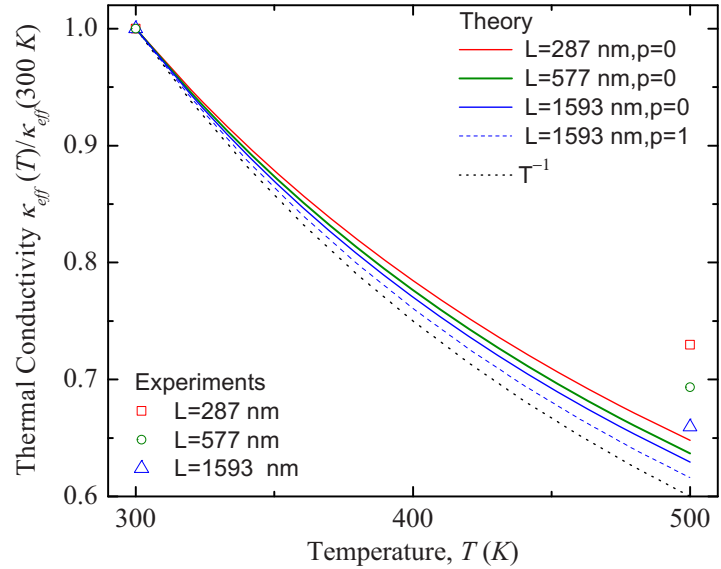

FIG. 9. (Color online) Temperature dependence of normalized effective lattice thermal conductivities for periods of 287,577 , and $1590 \mathrm{~nm}$. All thermal conductivities are normalized as $\kappa_{\text {eff }}(T) / \kappa_{\text {eff }}(300 \mathrm{~K})$.

ultrashort-period $\mathrm{Pb}_{2} \mathrm{Te}_{6} \mathrm{Sb}_{11}$ phase, this apparent agreement should be viewed with some caution because significant wave effects ${ }^{3,4}$ are expected at these length scales.

As shown in Fig. 8, for an annealing temperature of 773 $\mathrm{K}$ the normalized experimental data follow the same general trend as the model curves, although with a steeper reduction as the period is reduced below around $500 \mathrm{~nm}$. For a period of $\sim 500 \mathrm{~nm}$, the samples annealed at $673 \mathrm{~K}$ show a significantly lower thermal conductivity that those annealed at 773 $\mathrm{K}$. Although these points are lower than any of the model curves, this trend is qualitatively consistent with rougher interfaces (lower $p$ ), which might be expected due to the reduced atomic diffusivities at this lower annealing temperature.

To compare the temperature dependence of the model and experiment, Fig. 9 shows the thermal conductivities normalized to their values at $300 \mathrm{~K}$. As expected, for both model and experiment the trend is toward bulk behavior (Umklapp dominated: $\kappa \propto T^{-1}$ ) for increasing period and increasing specularity, because these conditions correspond to reduced importance of boundary scattering. In the opposite limit of small period, boundary scattering dominates, which in this regime of $T>\theta_{D}$ corresponds to $\kappa_{\text {eff }}$ independent of $T$ (recall Fig. 6). We note that the temperature dependence of the experimental data in Fig. 9 is somewhat weaker than any of the model curves. For example, the experimental results for $L$ $=287 \mathrm{~nm}$ imply $\kappa_{\text {eff }} \propto T^{-0.61}$. This suggests that the boundary scattering in the experiments is somewhat stronger than in the model, although this could also be related to the increased importance of the ambipolar effect at higher temperature.

\section{CONCLUSIONS}

We have established a model to calculate the effective thermal conductivity $\kappa_{\text {eff }}$ of a nanobulk material made of randomly oriented anisotropic superlattice grains. The analytical averaging rule of Mityushov and Adamesku ${ }^{22}$ was verified using FEM simulations. Calculations and sensitivity analysis show that $\kappa_{\text {eff }}$ of a nanobulk material is best understood by interpreting the grains as conductances in parallel, 
which for highly anisotropic grains is controlled by the maximum value of $\left(\kappa_{x}, \kappa_{z}\right)$; that is, by the "leakiest path."

Within each grain, the in-plane $\left(\kappa_{x}=\kappa_{y}\right)$ and cross-plane $\left(\kappa_{z}\right)$ thermal conductivities are found by solving the frequency-dependent BTE. For $\kappa_{z}$, previous work had been limited to frequency-independent (gray media) approximations, so a new solution methodology was developed to incorporate the frequency dependence of the group velocity and Umklapp scattering. By retaining the frequency dependence, this approach captures significant thermal conductivity reductions at larger periods than in the simpler graymedia calculations. For a $\mathrm{PbTe} / \mathrm{Sb}_{2} \mathrm{Te}_{3}$ nanobulk system, our model shows that $\kappa_{x}$ is four to five times larger than $\kappa_{z}$, indicating that efforts to manipulate $\kappa_{\text {eff }}$ should focus on engineering $\kappa_{x}$ rather than $\kappa_{z}$.

Experimental measurements of a $\mathrm{PbTe} / \mathrm{Sb}_{2} \mathrm{Te}_{3}$ nanobulk system also show the effect of short periods in reducing $\kappa_{\text {eff }}$, such as a $35 \%$ reduction in $\kappa_{\text {eff }}$ at a period $L=577 \mathrm{~nm}$ as compared to $L=1590 \mathrm{~nm}$. Comparison of the normalized thermal conductivity between model and experiments suggests that the effective specularity increases with increasing annealing temperature, and shows that these samples are in a mixed regime where both Umklapp and boundary scattering are important.

\section{ACKNOWLEDGMENTS}

This work is supported in part by the DARPA/DSO NMP program (Grant No. W911NF-08-C-0058) and the PRESTO program of Japan Science and Technology Agency. The views, opinions, and/or findings contained in this article are those of the authors and should not be interpreted as representing the official views or policies, either expressed or implied, of the Defense Advanced Research Projects Agency or the Department of Defense. Approved for Public Release, Distribution Unlimited.

\section{APPENDIX A: ASYMPTOTIC POWER LAWS OF FIG. 2}

We here give a physical and dimensional interpretation of the asymptotic power laws seen in Fig. 2. Consider first a material dominated by the physics of conductances in parallel, such as a thin film between parallel plates, and within each randomly-oriented grain let $\kappa_{x} \ll \kappa_{z}$. Clearly, when $\kappa_{x}$ $=0$ the heat transfer is still finite, due to the potential for parallel heat flow paths, and indeed the heat transfer must still depend on $\kappa_{z}$. In this case the general functional form $\kappa_{\text {eff,film }}=f\left(\kappa_{x}, \kappa_{z}\right)$ must reduce to a form

$$
\kappa_{\text {eff,film, } \infty}=f_{\infty}\left(\kappa_{z}\right) \text {, }
$$

where $f_{\infty}$ is a different function and the " $\infty$ " subscript indicates that this limit corresponds to $r \gg 1$. But every physical relation must be expressible in dimensionless form, and thus the only possible nondimensionalization of Eq. (A1) is

$$
\kappa_{\text {eff,film, }, \infty} / \kappa_{z}=c_{\infty}
$$

where $c_{\infty}$ must be a numerical constant. Nondimensionalizing this result yields

$$
\kappa_{\text {eff,film, }, \infty} / \kappa_{\text {char }}=c_{\infty} r^{2 / 3} \quad(r \gg 1),
$$

thus explaining the asymptotic behavior seen in Fig. 2 for the thin film. Similar reasoning in the limit $r \ll 1$ (subscript " 0 ") verifies

$$
\kappa_{\text {eff, film }, 0} / \kappa_{\text {char }}=c_{0} r^{-1 / 3} \quad(r \ll 1) .
$$

For a material such as a wire where the physics is dominated by resistances in series, the criteria for one of the principal conductivities to drop out of the problem is now that it is very large. Thus, in this case, when $\kappa_{x} \ll \kappa_{z}$ we note that it is now $\kappa_{z}$ that ceases to be a parameter, and the general form $\kappa_{\text {eff,wire }}=g\left(\kappa_{x}, \kappa_{z}\right)$ must now reduce to

$$
\kappa_{\text {eff }, \text { wire }, \infty}=g_{\infty}\left(\kappa_{x}\right) .
$$

Thus, applying the same arguments as the preceding paragraph, it is easy to show

$$
\kappa_{\text {eff,wire, },} / \kappa_{\text {char }} \propto r^{-1 / 3} \quad(r \gg 1),
$$

and

$$
\kappa_{\text {eff, wire }, 0} / \kappa_{\text {char }} \propto r^{2 / 3} \quad(r \ll 1),
$$

explaining the asymptotic behavior in Fig. 2 for a wire.

\section{APPENDIX B: ESTIMATING THE SOUND VELOCITIES OF $\mathrm{SB}_{2} \mathrm{TE}_{3}$}

Although the physical properties of $\mathrm{Sb}_{2} \mathrm{Te}_{3}$ are difficult to find in the literature, its close counterpart $\mathrm{Bi}_{2} \mathrm{Te}_{3}$ has been well studied. Thus, we estimate the sound velocities of $\mathrm{Sb}_{2} \mathrm{Te}_{3}$ indirectly from $\mathrm{Bi}_{2} \mathrm{Te}_{3}$ as follows.

First, the sound velocities of $\mathrm{Bi}_{2} \mathrm{Te}_{3}$ are calculated using continuum elasticity. ${ }^{50,51}$ For waves propagating in the $a$ direction of $\mathrm{Bi}_{2} \mathrm{Te}_{3}$, we calculate the longitudinal sound velocity $v_{s L}=\sqrt{C_{11} / \rho}=2884 \mathrm{~m} / \mathrm{s}$, where $C_{11}$ is an elastic stiffness constant and $\rho$ is the mass density. Following Ref. 51 two transverse sound velocities $v_{S T 1}=2170 \mathrm{~m} / \mathrm{s}$ and $v_{S T 2}$ $=1390 \mathrm{~m} / \mathrm{s}$ can also be obtained. Similarly, for waves propagating in the $c$ direction of $\mathrm{Bi}_{2} \mathrm{Te}_{3}$, we calculate $v_{s L}$ $=2539 \mathrm{~m} / \mathrm{s}$ and $v_{S T 1}=v_{s T 2}=1835 \mathrm{~m} / \mathrm{s}$. Then, we average these three velocities to obtain the average sound velocity by $v_{s, \text { avg }}=1 / 3\left(v_{s, L}+v_{s, T 1}+v_{s, T 2}\right)$. Thus we can obtain the average sound velocities of 2147 and $2070 \mathrm{~m} / \mathrm{s}$ along the $a$-plane and $c$-axis directions, respectively.

Next, to scale these results from $\mathrm{Bi}_{2} \mathrm{Te}_{3}$ to $\mathrm{Sb}_{2} \mathrm{Te}_{3}$, we note from elasticity theory that the sound velocity has the form $^{30} v_{s}=a \sqrt{2 \beta / M}$, where $a$ is the lattice constant, $\beta$ is a force constant, and $M$ is the total mass of all atoms in one primitive unit cell. Because $\mathrm{Bi}_{2} \mathrm{Te}_{3}$ and $\mathrm{Sb}_{2} \mathrm{Te}_{3}$ have the same crystal structure and bonding, we assume that their force constants are approximately equal, and thus estimate the sound velocities of $\mathrm{Sb}_{2} \mathrm{Te}_{3}$ using

$$
\frac{v_{s, \mathrm{Sb}_{2} \mathrm{Te}_{3}}}{v_{s, \mathrm{Bi}_{2} \mathrm{Te}_{3}}}=\frac{a_{\mathrm{Sb}_{2} \mathrm{Te}_{3}}}{a_{\mathrm{Bi}_{2} \mathrm{Te}_{3}}} \sqrt{\frac{M_{\mathrm{Bi}_{2} \mathrm{Te}_{3}}}{M_{\mathrm{Sb}_{2} \mathrm{Te}_{3}}} .}
$$

The corresponding velocity ratios in the $a$ and $c$ directions are 1.096 and 1.086 , respectively, which finally allows us to calculate the $\mathrm{Sb}_{2} \mathrm{Te}_{3}$ velocities reported in Table II. 
${ }^{1}$ T. C. Harman, P. J. Taylor, M. P. Walsh, and B. E. LaForge, Science 297, 2229 (2002).

${ }^{2}$ R. Venkatasubramanian, E. Siivola, T. Colpitts, and B. O'Quinn, Nature (London) 413, 597 (2001).

${ }^{3}$ M. V. Simkin and G. D. Mahan, Phys. Rev. Lett. 84, 927 (2000).

${ }^{4}$ B. Yang and G. Chen, Phys. Rev. B 67, 195311 (2003).

${ }^{5}$ G. Chen, ASME J. Heat Transfer 119, 220 (1997).

${ }^{6}$ G. Chen, Phys. Rev. B 57, 14958 (1998).

${ }^{7}$ D. L. Medlin and G. J. Snyder, Curr. Opin. Colloid Interface Sci. 14, 226 (2009).

${ }^{8}$ B. Poudel, Q. Hao, Y. Ma, Y. Lan, A. Minnich, B. Yu, X. Yan, D. Wang, A. Muto, D. Vashaee, X. Chen, J. Liu, M. S. Dresselhaus, G. Chen, and Z. Ren, Science 320, 634 (2008).

${ }^{9}$ Y. Ma, Q. Hao, B. Poudel, Y. Lan, B. Yu, D. Wang, G. Chen, and Z. Ren, Nano Lett. 8, 2580 (2008).

${ }^{10}$ Y. Lan, A. J. Minnich, G. Chen, and Z. Ren, Adv. Funct. Mater. 20, 357 (2010).

${ }^{11}$ G. J. Snyder and E. S. Toberer, Nature Mater. 7, 105 (2008).

${ }^{12}$ K. F. Hsu, S. Loo, F. Guo, W. Chen, J. S. Dyck, C. Uher, T. Hogan, E. K. Polychroniadis, and M. G. Kanatzidis, Science 303, 818 (2004).

${ }^{13}$ T. Ikeda, L. A. Collins, V. A. Ravi, F. S. Gascoin, S. M. Haile, and G. J. Snyder, Chem. Mater. 19, 763 (2007).

${ }^{14}$ C.-K. Liu, C.-K. Yu, H.-C. Chien, S.-L. Kuo, C.-Y. Hsu, M.-J. Dai, G.-L. Luo, S.-C. Huang, M.-J. Huang, and H. Mei-Jiau, J. Appl. Phys. 104, 114301 (2008).

${ }^{15}$ H. S. Carslaw and J. C. Jaeger, Conduction of Heat in Solids (Oxford University Press, London, 1959), p. 40.

${ }^{16}$ C. Chiritescu, D. G. Cahill, N. Nguyen, D. Johnson, A. Bodapati, P. Keblinski, and P. Zschack, Science 315, 351 (2007).

${ }^{17}$ W. Voigt, Lehrbuch der Krystallphysik (Teubener, Berlin, Germany, 1911).

${ }^{18}$ B. L. Adams and T. Olson, Prog. Mater. Sci. 43, 1 (1998).

${ }^{19}$ K. Schulgasser, J. Phys. C 10, 407 (1977)

${ }^{20}$ M. Avellaneda, A. V. Cherkaev, K. A. Lurie, and G. W. Milton, J. Appl. Phys. 63, 4989 (1988).

${ }^{21}$ V. Nesi and G. W. Milton, J. Mech. Phys. Solids 39, 525 (1991).

${ }^{22}$ E. A. Mityushov, R. A. Adamesku, and P. V. Gel'd, J. Eng. Phys. 47, 1052 (1984).

${ }^{23}$ W. H. Press, S. A. Teukolsky, W. T. Vetterling, and B. P. Flannery, Numerical Recipes: The Art of Scientific Computing, 3rd ed. (Cambridge University Press, Cambridge, 2007), p. 1130.

${ }^{24}$ N. W. Ashcroft and N. D. Mermin, Solid State Physics (Saunders College Publishing, New York, 1976).

${ }^{25}$ I. B. Mason and R. H. Knibbs, Nature (London) 198, 850 (1963).

${ }^{26}$ D. F. Pedraza and P. G. Klemens, Carbon 31, 951 (1993).
${ }^{27}$ C. Dames and G. Chen, in Thermoelectrics Handbook, Macro to Nano, edited by D. M. Rowe (Taylor \& Francis, New York, 2006).

${ }^{28}$ C. Dames and G. Chen, J. Appl. Phys. 95, 682 (2004).

${ }^{29}$ N. Mingo, Phys. Rev. B 68, 113308 (2003).

${ }^{30}$ C. Kittel, Introduction to Solid State Physics, 7th ed. (Wiley, New York, 1996), p. 100.

${ }^{31}$ B. Abeles, Phys. Rev. 131, 1906 (1963).

${ }^{32}$ Y.-J. Han, Phys. Rev. B 54, 8977 (1996).

${ }^{33}$ C. J. Glassbrenner and G. A. Slack, Phys. Rev. 134, A1058 (1964).

${ }^{34}$ O. Madelung, Semiconductors: Data Handbook, 3rd ed. (Springer, New York, 2004).

${ }^{35}$ D. Greig, Phys. Rev. 120, 358 (1960).

${ }^{36}$ E. Devyatkova, I. Smirnov, and F. T. Tela, Sov. Phys. Solid State 3, 1675 (1962).

${ }^{37}$ H. J. Goldsmid, Proc. Phys. Soc. London, Sect. B 69, 203 (1956).

${ }^{38}$ T. Caillat, M. Carle, P. Pierrat, H. Scherrer, and S. Scherrer, J. Phys. Chem. Solids 53, 1121 (1992).

${ }^{39} \mathrm{H}$. Scherrer and S. Scherrer, in CRC Handbook of Thermoelectrics, edited by D. M. Rowe (CRC, Cleveland, 1995).

${ }^{40}$ Z. Zhang, Nano/Microscale Heat Transfer (McGraw-Hill, New York, 2007), p. 274.

${ }^{41}$ J. M. Ziman, Electrons and Phonons: The Theory of Transport Phenomena in Solids (Oxford, New York, 1960), p. 459.

${ }^{42}$ S. M. Lee, D. G. Cahill, and R. Venkatasubramanian, Appl. Phys. Lett. 70, 2957 (1997).

${ }^{43}$ C. Dames, M. S. Dresselhaus, and G. Chen, in Thermoelectric Materials 2003 Research and Application, edited by G. S. Nolas, MRS Symposia Proceedings No. 793 (Materials Research Society, Pittsburgh, 2004), p. S1.2.

${ }^{44}$ T. Ikeda, V. Ravi, and G. Jeffrey Snyder, Metall. Mater. Trans. A 41, 641 (2010).

${ }^{45}$ T. Ikeda, V. A. Ravi, and G. J. Snyder, J. Mater. Res. 23, 2538 (2008).

${ }^{46}$ T. Ikeda, V. A. Ravi, L. A. Collins, S. M. Haile, and G. J. Snyder, J. Electron. Mater. 36, 716 (2007).

${ }^{47}$ T. Ikeda, E. S. Toberer, A. R. Vilupaur, S. M. Haile, and G. J. Snyder, in 26th International Conference on Thermoelectrics, edited by I.-H. Kim (IEEE, Jeju, Korea, 2007), pp. 1-4.

${ }^{48}$ L. Shelimova, O. Karpinskii, T. Svechnikova, E. Avilov, M. Kretova, and V. Zemskov, Inorg. Mater. 40, 1264 (2004).

${ }^{49}$ G. S. Nolas, in Thermal Conductivity: Theory, Properties, and Applications, edited by T. M. Tritt (Kluwer Academic, New York, 2004), p. 110.

${ }^{50}$ J. O. Jenkins, J. A. Rayne, and R. W. Ure, Phys. Rev. B 5, 3171 (1972).

${ }^{51}$ Y. C. Akgöz, G. A. Saunders, and Z. Sümengen, J. Mater. Sci. 7, 279 (1972). 